\title{
Recidiva de empiema subdural postquirúrgico
}

\author{
Y. Romero-Pizarro; M. Muñoz-Algarra*; C. Fernández-Mateos** e I. Sánchez-Romero*
}

Servicios de Medicina Interna, Microbiología* y Neurocirugía**. Hospital Puerta de Hierro. Majadahonda. Madrid.

\section{Resumen}

Se presenta un caso de recidiva de infección postquirúrgica en forma de empiema subdural por Proprionibacterium acnes tras un primer empiema drenado en el que no se llegó al diagnóstico microbiológico. $P$ acnes es un microorganismo gram positivo, anaerobio, que forma parte de la flora saprófita de la piel y de otras zonas del organismo. Sin embargo puede producir infecciones, entre otras localizaciones, en el sistema nervioso central (SNC), especialmente infecciones postquirúrgicas en las que puede llegar a ser el segundo germen en frecuencia después de Staphylococcus aureus. Es de crecimiento lento y suele crecer mejor en medios anaeróbicos líquidos. Suele ser resistente al metronidazol y sensible a penicilinas. En las infecciones postquirúrgicas del sistema nervioso central hay que tener en cuenta la posibilidad de este microorganismo, procesar las muestras de forma adecuada y mantenernos en contacto con el Servicio de Microbiología.

PALABRAS CLAVE. Proprionibacterium. Infección Postquirúrgica

Postoperative recurrence of subdural empyema

\section{Summary}

We present a case of recurrent subdural post-surgical empyema by Proprionibacterium acnes after a first drained empyema in which no microbiological diagnosis was reached. $P$. acnes is a gram-positive anaerobic organism which is part of the saprophytic flora of the skin and others parts of the body. However, it can cause infections, as in the central nervous system, especially post-surgical infections in which can be the second more frequent organism after Staphylococcus aureus. P. acnes grows slowly and shows better growth in liquid anaerobic media. It is usually resistant to metronidazol and

Recibido: 15-03-10. Aceptado: 6-12-10 sensitive to penicillin. In postoperative central nervous system infections we must take into account the possibility of this organism, process the sample properly and keep touch with the Microbiology Department.

KEY WORDS. Proprionibacterium. Postoperative infection

\section{Introducción}

Presentamos el caso de un varón previamente sano que desarrolla un empiema postquirúrgico de difícil diagnóstico tras la extirpación de un meningioma transicional.

\section{Caso clínico}

Se trata de varón de 74 años de edad que había sido intervenido de un meningioma transicional (grado I de la OMS) parasagital frontal izquierdo. Un mes después de esta cirugía consultó por infección de la herida quirúrgica que presentaba una zona central dehiscente por la que salía material purulento. El paciente no había tenido fiebre, su estado general era bueno y presentaba una exploración neurológica normal. Una resonancia magnética (RM) realizada en ese momento mostró, adyacente a la zona de la craniectomía, una colección subdural hipodensa, con burbujas en su interior, que se realzaba periféricamente tras las administración de gadolinio (fig. 1). Se realizó reapertura de craneotomía, saliendo colección purulenta epidural que se evacuó y envió para estudio microbiológico. Se inició antibioterapia empírica con ceftazidima y vancomicina intravenosas. Los cultivos bacterianos fueron negativos. El paciente fue dado de alta con tratamiento oral con ciprofloxacino y cotrimoxazol durante dos semanas.

Un mes y medio después del alta, el paciente fue llevado al servicio de urgencias por una crisis tónico-clónica generalizada. Había tenido febrícula los días previos sin otros datos clínicos de interés. A la exploración física presentaba buen estado general, estaba afebril, tenía un Glasgow de 15/15, no mostraba focalidad neurológica y la herida quirúrgica no presentaba datos de infección. 


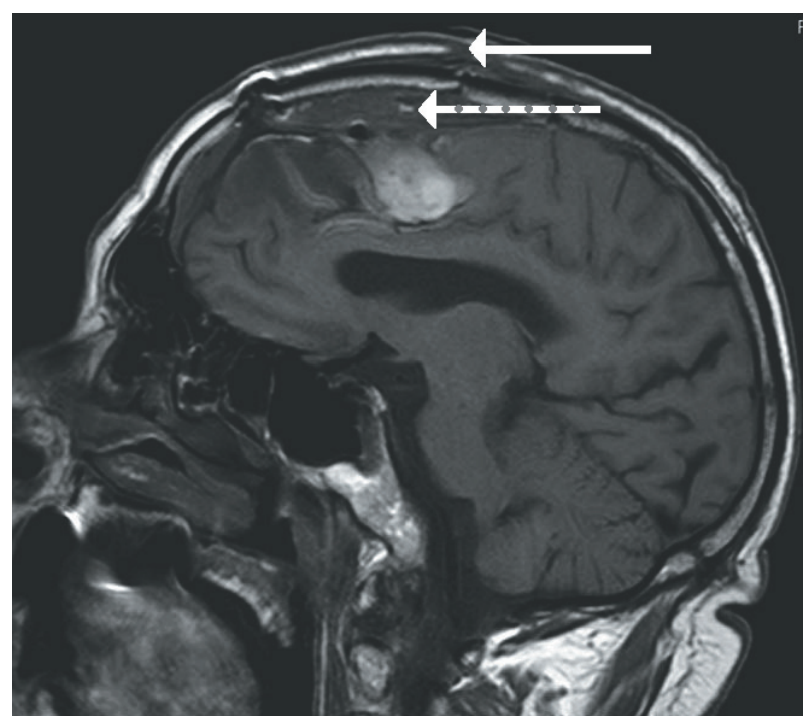

Figura 1. Imagen de RNM con gadolinio: subyacente a la craneotomía (flecha contínua) existe una colección extraparenquimatosa con algunas burbujas de aire (flecha discontínua).

Se inició tratamiento anticonvulsivante y se realizó una tomografía computarizada que puso de manifiesto una colección subdural de predominio hipodenso, con realce periférico tras administración de contraste y un margen interno convexo hacia el parénquima cerebral. Persistía aire en el interior de la colección.

Se inició tratamiento antibiótico empírico con ceftazidima y vancomicina. Con la sospecha diagnóstica de recidiva de empiema subdural se realizó una tercera craniectomía que puso de manifiesto una colección subdural de aspecto purulento que se evacuó. Se enviaron muestras para microbiología del material drenado y de una pieza de craniectomía adyacente a la colección que se extirpó con fines terapéuticos por eventual infección (fig. 2) y se embebió en caldo de tioglicolato para su procesamiento.

A los 5 días de incubación se constató el crecimiento de Proprionibacterium acnes (P. acnes) tras subcultivo en medio sólido y en atmósfera de anaerobiosis del caldo de enriquecimiento. Se realizó antibiograma mediante E-test encontrándose resistencia a metronidazol y sensibilidad a penicilina, amoxicilina-clavulánico, ceftazidima, clindamicina y vancomicina. Se suspendió el tratamiento con vancomicina y ceftazidima iniciándose tratamiento con amoxicilina-clavulánico. La evolución del paciente fue buena y fue dado de alta con amoxicilina oral durante un mes y el diagnóstico de "recidiva de empiema subdural postquirúrgico por Propionibacterium acnés".

\section{Discusión}

Propionibacterium acnes es un bacilo grampositivo
2011; 22: 264-266

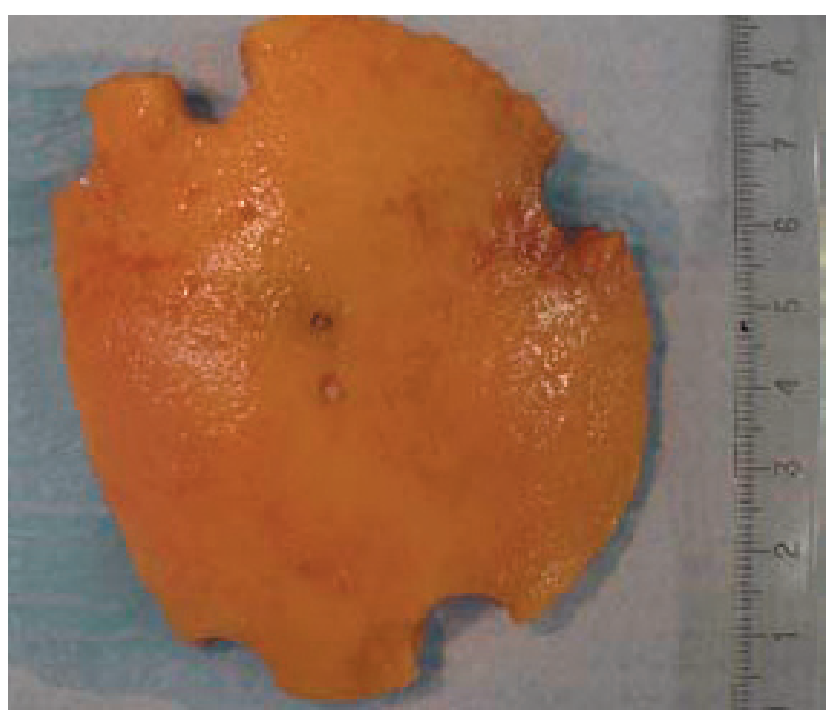

Figura 2. Pieza de craniectomía extirpada previa a la evacuación de segundo empiema subdural.

pleomorfo, anaerobio y aerotolerante, no esporulado e inmóvil, que actualmente se incluye en la familia Actinomycetaceae. Forma parte de la flora saprófita de la piel, nasofaringe, cavidad oral, tracto intestinal y genitourinario $^{8}$ aislándose de forma característica de los folículos sebáceos y lesiones de acné vulgaris. Habitualmente ha sido considerado un microorganismo contaminante de los distintos medios de cultivo por su escaso poder patógeno, aunque puede ser responsable de infecciones graves. Están bien documentadas las infecciones del sistema nervioso central (SNC) generalmente asociadas a la colocación de derivaciones intracraneales ${ }^{1,3,4,5,9}$. También se ha implicado en infecciones de prótesis cardíacas y ortopédicas, de heridas quirúrgicas, en abscesos cutáneos, conjuntivitis en pacientes portadores de lentes de contacto, e incluso en infecciones crónicas de los discos lumbares vertebrales de pacientes con ciática grave $\mathrm{e}^{3,12,13}$.

Los factores de riesgo para infección por $P$. acnes son la presencia de cuerpos extraños (válvulas de derivación ventricular, drenajes ventriculares, prótesis, etc), traumatismos penetrantes, cirugía previa, diabetes, y la existencia de inmunosupresión ${ }^{3}$.

$P$. acnes es un microorganismo de crecimiento lento que necesita 4-7 días para crecer y algunos autores recomiendan incubar hasta 14 días en medio líquido ${ }^{11}$ ya que este microorganismo crece más rápidamente en medios anaeróbicos líquidos que en medios sólidos ${ }^{2}$

Una característica de este microorganismo anaerobio es su resistencia al metronidazol, como se demuestra en nuestro aislado, manteniendo la sensibilidad a las grandes familias de antibióticos utilizadas en infecciones por anaerobios (penicilinas, carbapenémicos y clindamicina). 
La sensibilidad que muestran a la eritromicina, tetraciclinas y cefalosporinas es variable ${ }^{10}$.

Para atribuir la etiología de una infección a $P$. acnes algunos autores recomiendan, en los casos y patologías en que sea posible, la realización de 4 muestras profundas para facilitar la interpretación de los cultivos².

A pesar de que la infección del sistema nervioso central después de procedimientos neuroquirúrgicos es poco frecuente (en las grandes series se sitúa alrededor del $1 \%{ }^{6}$ ) es una causa importante de morbimortalidad para estos pacientes. Entre los patógenos más frecuentemente implicados en el empiema subdural se encuentran Staphylococcus spp, Streptococcus spp y microorganismos anaerobios ${ }^{6}$. Entre estos últimos, $P$. acnes ha demostrado ser un agente prevalente en este tipo de infecciones originando en algunas series ${ }^{6}$ hasta el $25 \%$ de las mismas ocupando el segundo lugar después de Staphylococcus aureus.

El diagnóstico de las infecciones postquirúrgicas, generalmente, no plantea dificultades debido a la relación temporal con la cirugía y a los síntomas de fiebre, síndrome confusional, focalidad neurológica, etc. Sin embargo, se requiere un alto índice de sospecha en el caso de las infecciones por $P$. acnes que tienen con frecuencia un curso indolente y subagudo que puede dificultar el diagnóstico $^{1,5,7}$.

Los hechos comentados, especialmente el curso indolente de la infección por P. acnes y la dificultad para su aislamiento, hacen más frecuente un infradiagnóstico de estas infecciones. Un ejemplo de este infradiagnóstico puede ser el primer empiema del caso que comentamos, en el que, la no identificación del microorganismo, pudo condicionar la recidiva del empiema. El cultivo en medio líquido de la pieza de craniectomía facilitó el crecimiento del P. acnes en la segunda ocasión y el adecuado tratamiento antibiótico del paciente.

\section{Conclusiones}

Concluimos, por tanto que, en casos de infecciones del SNC postquirúrgicas se debe tener muy en cuenta la posibilidad de infección por P. acnes, por lo que las muestras para estudio microbiológico deben ser remitidas lo más rápidamente posible $\mathrm{y}$ en medio de trasporte específico para microorganismos anaerobios al Servicio de Microbiología, e informar al Microbiólogo para que se realicen los cultivos en las condiciones adecuadas y se mantengan el tiempo suficiente como para poder descartar su crecimiento. También debe ser tenido en cuenta este microorganismo a la hora de plantear el tratamiento empírico de un empiema postquirúrgico antes de tener los resultados de los cultivos.

\section{Bibliografía}

1. Barazi, S.A., Gnanalingham, K.K., Chopra, I., Van Dellen, J.R.: Delayed postoperative intracerebral abscess caused by Proprionibacterium acnes: case report and review of the literature. Br J Neurosurg. 2003; 17: 336-339.

2. Bemer, P., Corvec, S., Tariel, S., et al.: Significance of Propionibacterium acnes-positive samples in spinal instrumentation. Spine 2008; 33: E971-976.

3. Brook, I., Frazier, E.H.: Infections caused by Propionibacterium species. Rev Infect Dis. 1991;13: 819-822.

4. Critchley, G., Strachan, R.: Postoperative subdural empyema caused by Propionibacterium acnes - a report of two cases. Br J Neurosurg 1996; 10: 321-323.

5. Ghalayini, S.R.A., Likhith, A.M., Golash, A.: Propionibacterium acnes causing delayed subdural empyema -a case report and review of literature. J Clin Neuroscience 2004; 11 : 677-679.

6. Hearwood, M.C., Walter, A.H.: Postoperative central nervous system infection: incidence and associated factors in 2111 neurosurgical procedures. Clin Inf Dis 2007; 45: 55-59

7. Kim, J.H., Lee, C.H., Hwang, S.H., Kang, D,H.: Superimposed propionibacterium acnes subdural empyema in a patient with chronic subdural hematoma. J Korean Neurosurg Soc. 2009 Jan; 45: 53-56.

8. Könönen, E., Wade, W.G.: Propionibacterium, Lactobacillus, Actinomyces, and other non-spore-forming anaerobic Gram-positive rods En: Murray PR, Baron EJ, Jorgensen JH, Landry ML, Pfaller MA editors. Manual of Clinical Microbiology. $9^{\text {th }}$ ed. Washington: ASM press; 2007. p. 872-88.

9. Lalueza, A., Díaz-Pedroche, C., Broseta, A., San Juan, R.: Empiema subdural subagudo. Enferm Infecc Microbiol Clin 2005; 23: 381-382.

10. Mascini, E.M., Verhoef, J.: Anaerobic gram-positive nonsporulating bacilli. En: Mandell GL, Bennett JE, Dolin $\mathrm{R}$ (ed). Principles and practice of infectious diseases. $5^{\text {th }}$ ed. Philadelphia: Churchill Livingstone; 2000. p. 2573-5

11. Meredith, F.T., Phillips, H.K., Reller, L.B.: Clinical utility of broth cultures of cerebrospinal fluid from patients at risk for shunt infections. J Clin Microbiol 1997; 35: 3109-3111

12. Stirling, A., Worthington, T., Rafiq, M., Lambert, P.A., Elliott, T.S.J.: Association between sciatica and Propionibacterium acnes. Lancet 2001; 357 (9273): 2024

13. Tunney, M.M., Patrick, S., Gorman, S.P. et al.: Improved detection of infection in hip replacements. A currently underestimated problem. J Bone Joint Surg Br 1998; 80: 568-572.

Romero-Pizarro, Y.; Muñoz-Algarra, M.; FernándezMateos, C.; Sánchez-Romero, I.: Recidiva de empiema subdural postquirúrgico. Neurocirugía 2011; 22: 264-266. 